\title{
TRABALHO NA ESTRATÉGIA DA SAÚDE DA FAMÍLIA: IMPLICAÇÕES NAS CARGAS DE TRABALHO DE SEUS PROFISSIONAIS*
}

Letícia de Lima Trindade', Denise Elvira Pires de Pires², Simone Coelho Amestoy¹, Elaine Cristina Novatzki Forte ${ }^{3}$, Francele Luz Machado ${ }^{4}$, Maiara Bordignon ${ }^{4}$

'Enfermeira. Doutora em Enfermagem. Universidade do Estado de Santa Catarina. Chapecó-SC-Brasil. ${ }^{2}$ Enfermeira. Doutora em Ciências Sociais. Universidade Federal de Santa Catarina. Florianópolis-SC-Brasil. ${ }^{3}$ Enfermeira. Mestre em Enfermagem. Universidade Federal de Santa Catarina. Florianópolis-SC-Brasil. ${ }^{4}$ Acadêmica de Enfermagem. Universidade Federal de Santa Catarina. Florianópolis-SC-Brasil.

RESUMO: Objetivou-se identificar aspectos do trabalho na Estratégia de Saúde da Família que contribuem para aumentar e/ou reduzir as cargas de trabalho dos profissionais. Participaram 11 profissionais de três equipes de Estratégia de Saúde da Família de um estado do Sul do Brasil. Os dados foram coletados, entre dezembro de 2010 e março de 2011, por meio de entrevistas individuais, grupo focal e estudo documental e analisados combinando a Análise Temática com recursos do software ATLAS.ti. As principais fontes de aumento das cargas foram aspectos que impedem a implantação do modelo assistencial como preconizado; e de redução, a afinidade com o modelo assistencial, a autonomia da equipe e a garantia do emprego. Predominaram, no trabalho da Estratégia de Saúde da Família, fragilidades que repercutem negativamente na satisfação e na saúde dos profissionais, mas há possibilidades de intervenção para redução das cargas e qualificação da assistência, especialmente aquelas para melhorias das condições de trabalho.

DESCRITORES: Saúde do trabalhador; Carga de trabalho; Saúde da família; Atenção primária à saúde.

\section{WORKING IN THE FAMILY HEALTH STRATEGY: IMPLICATIONS IN PROFESSIONALS WORKLOADS}

\begin{abstract}
This study aimed to identify aspects of work in the Family Health Strategy that contribute to increase and / or reduce workloads of professionals. Eleven professionals from three teams of the Family Health Strategy from a state of southern Brazil participated in the study. Data were collected between December 2010 and March 2011, through individual interviews, focus groups and documentary study and were analyzed combining thematic analysis with the resources of the software ATLAS.ti. The main sources of increased workloads were aspects that hinder the implementation of the care model as recommended; and of reduced workloads, the affinity with the care model, the autonomy of the team and the guarantee of employment. In the work of the Family Health Strategy, some weaknesses that negatively affect satisfaction and health professionals predominated, but there are possibilities for intervention to reduce workloads and improve quality of care, especially those for better working conditions.
\end{abstract}

DESCRIPTORS: Occupational Health; Workload; Family health; Primary health care.

\section{TRABAJO EN LA ESTRATEGIA DE LA SALUD DE LA FAMILIA: IMPLICACIONES EN LAS CARGAS DE TRABAJO DE SUS PROFESIONALES}

RESUMEN: Fue objetivo del estudio identificar aspectos del trabajo en la Estrategia de Salud de la Familia que contribuyen para aumentar y/o reducir las cargas de trabajo de los profesionales. Participaron 11 profesionales de tres equipos de Estrategia de Salud de la Familia de un estado del Sur de Brasil. Los datos fueron obtenidos entre diciembre de 2010 y marzo de 2011, por medio de entrevistas individuales, grupo focal y estudio documental, siendo analizados mesclándose el Análisis Temático con recursos del software ATLAS.ti. Las principales fuentes de aumento de las cargas fueron aspectos que impiden la implantación del modelo asistencial como preconizado; y de reducción, la afinidad con el modelo asistencial, la autonomía del equipo y la garantía del empleo. Predominaron, en el trabajo de la Estrategia de Salud de la Familia, fragilidades que repercuten negativamente en la satisfacción y en la salud de los profesionales, pero hay posibilidades de intervención para reducción de las cargas y cualificación de la asistencia, de modo especial aquellas para mejorías de las condiciones de trabajo. DESCRIPTORES: Salud del trabajador; Carga de trabajo; Salud de la familia; Atención primaria a la salud.

* Artigo originado da tese de doutorado Implicações de dois modelos assistenciais nas cargas de trabalho dos profissionais de saúde da atenção básica. Universidade Federal de Santa Catarina, 2011. 


\section{INTRODUÇÃO}

Modelo assistencial em saúde compreende a organização de ações, que se articulam com recursos físicos, tecnológicos e humanos, para intervir no processo saúde-doença, enfrentar e resolver os problemas de saúde dos indivíduos e coletividades ${ }^{(1)}$. Constitui-se por diálogos e princípios que refletem uma determinada conjuntura epidemiológica e certo desenho de aspirações sobre o viver saudável (2).

Nesse cenário, a Estratégia de Saúde da Família (ESF) é um modelo assistencial em desenvolvimento no Brasil, desde 1994. Esta tem sido reconhecida como a principal intervenção para reorientação da Atenção Básica (AB), por buscar aproximar-se dos preceitos do Sistema Único de Saúde (SUS), e por primar pelo acesso universal e contínuo aos serviços de saúde de qualidade e resolutivos, a partir da descrição do território, e assim, planejamento e programação descentralizada ${ }^{(3)}$.

Esse novo modelo preconizado pelo Ministério da Saúde Brasileiro (MS) prevê um olhar para a coletividade, tendo como foco a família e a assistência multiprofissional. Estudos visualizam a ESF como uma estratégia que fortalece a $A B$ e a promoção da saúde, como via capaz de melhorar o status de saúde e reduzir custos ${ }^{(3-6)}$.

Entende-se que as características do processo de trabalho nesse modelo têm implicações na satisfação/insatisfação e nas cargas de trabalho das equipes, compreendidas como elementos do trabalho que interatuam entre si e com o corpo do trabalhador, podendo desencadear alterações biopsíquicas, possíveis de manifestação nos desgastes e adoecimento dos profissionais ${ }^{(7)}$. Assim, as cargas de trabalho têm forte influência das condições laborais e interferem diretamente no processo de desgaste do trabalhador, o que pode resultar em adoecimento ${ }^{(8-9)}$.

Nesse sentido, este estudo buscou identificar os aspectos/características do trabalho na ESF que aumentam e que reduzem as cargas de trabalho de seus profissionais.

\section{MÉTODO}

Trata-se de um estudo qualitativo, desenvolvido na $7^{a}$ Regional de Saúde, localizada na Região Sudoeste do Paraná, no Sul do Brasil.
A população da pesquisa foi composta por amostragem intencional, incluindo equipes referenciadas pela chefia da $7^{a}$ Regional de Saúde (53 equipes distribuídas entre 17 municípios), por desempenharem um trabalho de destaque no que se refere à qualidade da assistência prestada, no sentido de aproximação do que é preconizado pelo MS. Foram incluídas três equipes de ESF, totalizando 11 profissionais de saúde, sendo: 3 médicos(as), 3 enfermeiras, 3 técnicas de enfermagem, 1 dentista e 1 auxiliar de consultório odontológico.

Para a coleta de dados, realizada no período de dezembro de 2010 a março de 2011, foi utilizada a triangulação de instrumentos envolvendo estudo documental, entrevista individual semiestruturada e grupo focal. Buscou-se: identificar o cenário político-social e institucional onde o trabalho das equipes era realizado e a composição das mesmas; caracterizar as práticas assistenciais, a organização e divisão do trabalho nas equipes; e identificar as características do modelo assistencial da Saúde da Família em sua relação com as cargas de trabalho dos profissionais que compõem as equipes.

O processo de coleta de dados foi iniciado somente após a autorização das Secretarias Municipais de Saúde envolvidas; aprovação do Comitê de Ética em Pesquisa da Universidade Federal de Santa Catarina (n. 971/2010) e seguiu os aspectos éticos preconizados pela Resolução do Conselho Nacional de Saúde.

O estudo documental permitiu identificar as normas/rotinas das instituições, prontuário, caracterização da força de trabalho, da política salarial, divisão e formas de organização e gestão.

As entrevistas foram pré-agendada com os participantes, precedidas de esclarecimentos acerca da pesquisa e realizadas no local de trabalho, em espaço e horário escolhidos pelos entrevistados de modo a não intervir na dinâmica das atividades das unidades. $\mathrm{Na}$ ocasião das entrevistas foi solicitada a anuência de cada participante e a assinatura do termo de consentimento livre e esclarecido. As entrevistas buscaram caracterizar o trabalho dos profissionais de saúde, o modelo assistencial da ESF e sua influência nas cargas de trabalho.

Para aprofundar os achados, foi realizado um grupo focal, em três sessões de aproximadamente uma hora e meia cada, orientadas por um 
roteiro semiestruturado. As sessões do grupo focal ocorreram após o término das reuniões de equipe. Participaram das sessões entre cinco a dez participantes, nas quais foram discutidos temas como: trabalho na ESF; fontes de (in)satisfação, elementos que contribuem para redução e aumento das cargas de trabalho. O número de entrevistas e de sessões dos grupos focais foi considerado satisfatório, conforme saturação dos dados.

As informações coletadas foram organizadas com auxílio do software Atlas.ti e analisadas a partir da Análise Temática de Conteúdo ${ }^{(10)}$, emergindo duas categorias centrais, as fontes de aumento e fontes de redução das cargas de trabalho ${ }^{(7)}$.

\section{RESULTADOS}

Os participantes tinham, em média, 32 anos de idade (entre 24 e 39 anos); 9 do sexo feminino, com uma média de 8 anos de experiência na profissão e 3,5 anos na ESF. Ainda, três profissionais possuíam ensino médio completo, um ensino superior incompleto e sete com ensino superior. Em relação à formação no âmbito de pós-graduação, dois profissionais tinham especialização em saúde da família e dois na área clínico-cirúrgica.

Todos os profissionais possuíam contrato de 40 horas semanais, contudo 54,54\% possuíam outro emprego. Entre as atividades complementares, destacaram-se: consultório particular; atividade docente; atividade na área hospitalar ou em pronto atendimento. A média semanal de horas trabalhadas foi de 68 horas, variando de 40 horas até 76 horas semanais.

No Quadro 1 encontram-se as categorias que emergiram como fontes de aumento e de diminuição das cargas de trabalho na ESF.

Quadro 1 - Fontes de aumento e diminuição das cargas de trabalho na Estratégia de Saúde da Família. Paraná, 2011

\begin{tabular}{|l|l|}
\hline Fontes de aumento das cargas na ESF & Fontes de diminução das cargas na ESF \\
\hline Excesso de demanda & O próprio modelo assistencial da ESF \\
\hline Falta de preparo dos profissionais & O trabalho em equipe \\
\hline $\begin{array}{l}\text { Ausência do acolhimento ou a implantação deficitária } \\
\text { deste }\end{array}$ & $\begin{array}{l}\text { A proximidade dos profissionais com a realidade dos } \\
\text { usuários e famílias }\end{array}$ \\
\hline Foco na consulta médica & A presença do agente comunitário de saúde \\
\hline Agressões verbais por parte dos usuários & $\begin{array}{l}\text { A organização do trabalho com atividades agendadas } \\
\text { previamente }\end{array}$ \\
\hline $\begin{array}{l}\text { Falta de compreensão dos usuários e gestores acerca do } \\
\text { modelo assistencial }\end{array}$ & A flexibilidade na organização do trabalho \\
\hline $\begin{array}{l}\text { Fragilidade no estabelecimento de vínculo entre } \\
\text { profissionais e usuários }\end{array}$ & O vínculo empregatício por concurso público \\
\hline Contato cotidiano com a violência urbana & Menor exposição às cargas biológicas. \\
\hline $\begin{array}{l}\text { Falhas nos serviços de referência e ausência da } \\
\text { contrarreferência }\end{array}$ & \\
\hline $\begin{array}{l}\text { Fragilidade na gestão e forte influência políticopartidária } \\
\text { no cotidiano dos serviços }\end{array}$ & \\
\hline $\begin{array}{l}\text { Falta de incentivos e investimentos na qualificação dos } \\
\text { profissionais }\end{array}$ & \\
\hline $\begin{array}{l}\text { Insatisfação com salário, carga horária de trabalho, e } \\
\text { com a ausência do plano de cargos e salários }\end{array}$ & \\
\hline Problemas no relacionamento interpessoal na equipe & \\
\hline Déficits na estrutura física das unidades & \\
\hline $\begin{array}{l}\text { Divisão desigual do quantitativo de atividades entre os } \\
\text { membros da equipe. }\end{array}$ & \\
\hline
\end{tabular}


Os achados revelam os principais aspectos mencionados como causadores de aumento das cargas, com destaque para o excesso de demanda em face das condições de estrutura física das unidades, do número e da capacitação das equipes para atender às necessidades apresentadas pelos usuários.

Os aspectos destacados para o aumento das cargas de trabalho se relacionam com a forma de organização do trabalho das equipes e ao distanciamento desta do prescrito para o novo modelo assistencial ESF. Tal como descrito a seguir:

\section{[...] não é fácil porque a lógica da população ainda} é voltada para a consulta médica, eles querem passar pelo médico, sair com o remedinho e a receita embaixo do braço, essa é a parte difícil de romper. (Grupo Focal)

Também assim a falta de contrarreferência [...] você faz todo um acompanhamento daquele usuário, encaminha para um especialista, uma especialidade e não retorna a contrarreferencia e eu não sei o que fazer [...] não tenho feedback, isto me deixa muito cansada, muito irritada, é como se fosse uma ponte inacabada. (Enfermeira 2)

Entende-se que as agressões verbais dos usuários têm relação com as dificuldades destes e dos gestores entenderem o significado do novo modelo assistencial da ESF, de modo que dificulta a implantação do modelo preconizado, como por exemplo, no seguinte discurso:

\section{[...] o que dificulta é a falta de conhecimento das} famílias sobre o que é a ESF, eles sabem que aqui tem o posto, mas eles não sabem que esse posto visa promover a saúde e prevenir [doenças], eles ainda têm a visão curativista [...]. (Enfermeira 3)

A assistência às famílias em situação de violência doméstica, indivíduos marginalizados, expostos às drogas lícitas e ilícitas, por vezes com precárias condições de vida, elevam os riscos à sua saúde pela exposição desses profissionais às situações de violência urbana e de intenso desgaste, tal como verificado nos prontuários dos usuários das unidades pesquisadas.

Destacaram-se, ainda características relacionadas às condições inadequadas de trabalho, como: baixo salário (incoerente com as responsabilidades assumidas e com a produtividade das equipes); ausência do plano de cargos e salários e jornada de 40 horas semanais. Uma fala com esta característica foi:

O salário é ruim, o que aumenta muito a carga, a gente sai daqui muito cansado, porque são muitas pessoas, muito contato, muita demanda e pouco reconhecimento. (ACD 1)

Os conflitos interpessoais na equipe de trabalho, a escassez de recursos materiais, a má divisão do trabalho e quando esse é muito burocrático, têm repercussão no aumento das cargas. Destacou-se também o déficit de capacitação dos profissionais para trabalhar nesse modelo assistencial e a falta de incentivos para o aprimoramento por meio de pós-graduação.

Identificaram-se fragilidades na gestão e forte influência político-partidária nos processos decisórios, na rotatividade de profissionais nos cargos comissionados, na falta de continuidade de projetos e ações durante e após os processos eleitorais. Ainda, ausência de instrumentos de trabalho, em especial do prontuário eletrônico, entendido como útil para o melhor diálogo entre os serviços.

Os profissionais mencionaram a falta de protocolos assistenciais e o acesso restrito e truncado aos serviços hospitalares, o qual é reduzido e permeado pelo mix públicoprivado na região, além da escassez de serviços específicos.

Em relação às fontes de redução das cargas de trabalho, o principal motivo foi a identidade dos profissionais com o modelo prescrito para a ESF. Entre os aspectos da ESF identificados como facilitadores do trabalho estão a implantação do acolhimento, trabalho em equipe, proximidade com a realidade dos usuários e famílias e a presença dos agentes. Um relato trata deste aspecto:

[...] é que, assim, na residência a filosofia da medicina de família é muito bacana, [...] é um trabalho diferente baseado na prevenção e na promoção de saúde, você vai além da saúde, 
vê os fatores que interferem na saúde [...] essa comunidade tem esporte, tem o lazer, tem uma creche, tem onde trabalhar, isso tudo é saúde. (Médico 2)

A proximidade com a realidade das famílias e usuários, por meio da descrição da clientela, da visita domiciliar e o fortalecimento do elo entre equipes e famílias, especialmente pelo trabalho dos ACS, contribuem para a redução das cargas de trabalho.

Na organização da assistência, grande parte dos profissionais ressaltou que o agendamento dos atendimentos favorece a divisão equitativa do trabalho entre os membros da equipe e o planejamento mais eficiente das ações, reduzindo as cargas físicas e psíquicas. Os entrevistados enfatizaram a importância da autonomia para organizar o trabalho com certa flexibilidade, o que potencializa a criatividade profissional, bem como, os direitos trabalhistas e a garantia de emprego oportunizada pelo concurso público, são identificados como fonte de satisfação. O relato a seguir tem este teor:

Ser concursada me dá segurança para brigar pelo que eu acho justo. (Enfermeira 3)

Destacou-seo menor número de procedimentos técnicos invasivos em relação a outras realidades laborais, o que, consequentemente, gera menos exposição às cargas biológicas.

Os sujeitos apontaram aspectos/estratégias, não presentes na realidade atual, que poderiam reduzir o desgaste, entre eles: melhor divulgação do modelo assistencial; investimentos na qualificação profissional; organização da assistência mesclando o atendimento agendado com livre demanda; implantação do prontuário eletrônico, de um plano de cargos e salários condizente com as especificidades do modelo assistencial, incluindo incentivos à qualificação e redução da jornada de trabalho.

\section{DISCUSSÃO}

Ao analisar o perfil dos sujeitos da pesquisa, evidenciou-se que a amostra estudada é composta por adultos jovens. O pouco tempo de experiência profissional também foi encontrado em outro estudo como um fator que interfere na disponibilidade de recursos para o enfrentamento das situações que elevam o desgaste no trabalho na $\mathrm{ESF}^{(11-12)}$.

Constatou-se que os aspectos/características, que contribuíram, de modo mais significativo implicam, predominantemente, no aumento das cargas fisiológicas, psíquicas e cognitivas dos profissionais de saúde pesquisados. Entre as múltiplas dificuldades para trabalhar nesse novo modelo, o excesso de demanda se destaca e fragiliza a humanização da assistência nesses serviços $^{(13)}$. Além disso, o foco da demanda para a consulta médica interfere nas possibilidades de mudança na organização do trabalho e dificulta a implementação das diretrizes propostas pela ESF.

Outro aspecto verificado foi o efeito da formação inadequada dos profissionais, dificultando o desenvolvimento de práticas que atendam aos desafios presentes no momento da implementação do SUS e da ESF, acarretando aumento das cargas cognitivas e psíquicas.

Considerando-se o conjunto das equipes, verificou-se que apenas $18,2 \%$ da amostra tiveram alguma capacitação em nível de pósgraduação para atuar nesse modelo assistencial. Contudo, também deve haver preocupação com a reprodução nas escolas, da formação que prestigia o conhecimento restritamente especialista $^{(14)}$.

Entende-se que a dificuldade de implantação e consolidação das equipes também tem relação com o processo formativo, o qual permite pouco acesso ou acesso limitado aos serviços da atenção básica. Muitos profissionais de saúde têm recebido uma formação ainda associada à medicina privada, ao ambiente hospitalar e à negação dos aspectos não biológicos que interferem na saúde ${ }^{(14-16)}$. Nesse sentido, a ESF deve construir bases para o desenvolvimento de novas práticas sanitárias, com foco no vínculo, acolhimento e cuidado no contexto de uma atenção sanitária humanizada e humanística ${ }^{(3-6,15,17)}$.

As mudanças nos processos produtivos, em termos técnicos, organizacionais, políticos e culturais, tiveram marcante impacto sobre a formação profissional e exigem aprendizagem permanente ${ }^{(16-17)}$. Contudo, no cenário brasileiro, a adoção de políticas públicas para qualificação como direito é algo recente e se encontra em um estágio ainda tímido de formulação e implementação. O MS tem formulado diversas 
estratégias para promover a qualificação profissional na ESF, destacando-se a Universidade do SUS, o Pet-Saúde, entre outros ${ }^{(17)}$. Estas estratégias podem contribuir para melhor qualificação do trabalho e enfrentamento dos problemas vivenciados pelas equipes.

Em relação às condições de trabalho, identificou-se que a insatisfação com o salário e a excessiva carga horária de trabalho aumentam as cargas psíquicas, contribuindo para o desgaste. Diferentes categorias profissionais da saúde já conquistaram em Lei a regulamentação da jornada de trabalho e debatem a sua implementação nos serviços. Neste cenário destaca-se a luta dos profissionais de enfermagem pela aprovação na Câmara Federal do Projeto de Lei 2295/2000, que regulamenta a jornada de trabalho da categoria em 30 horas semanais ${ }^{(18)}$.

Cabe, ainda, questionar o tamanho da equipe mínima. Os achados revelam que as responsabilidades assumidas são incompatíveis com o número de profissionais disponíveis para a garantia da qualidade da assistência, aumentando as cargas fisiológicas e psíquicas. Uma estratégia poderia ser a ampliação da equipe mínima, no entanto o problema é complexo, o que demanda a busca de soluções criativas com vistas a potencializar a promoção da saúde, a articulação de ações de forma integral e focada na oferta do cuidado integral e humanizado.

Em relação às dificuldades na organização do trabalho para consolidação da ESF, os resultados identificam a necessidade da implantação do acolhimento, o qual prevê um aumento da resolutividade dos serviços, a ampliação do acesso, redução da demanda e a melhora na satisfação dos usuários ${ }^{(19-20)}$. Cabe retomar que o reconhecimento do trabalho da equipe destacouse como fonte de redução da carga de trabalho na realidade pesquisada.

Contudo, são muitos os entraves no exercício do acolhimento ${ }^{(13)}$, entre eles o descompasso entre demanda e recursos disponíveis, problemas em relação à organização do trabalho, que têm predominantemente, foco no atendimento médico. Esses achados sugerem a permanência de fortes traços do modelo biomédico no trabalho das equipes de ESF pesquisadas. Entretanto, os sujeitos do estudo se mostraram cientes da necessidade de redirecionamento das ações ofertadas, com o propósito de adequá-las à melhor escuta dos usuários, com estímulo à maior autonomia dos indivíduos, observando suas necessidades individuais e coletivas e resgate do trabalho interdisciplinar.

Os diversos problemas evidenciados na rede assistencial mencionados pelas equipes, certamente têm influência na qualidade dos serviços da $A B$, prejudicam a consolidação da ESF e elevam, especialmente, as cargas de trabalho fisiológicas e psíquicas dos profissionais. Esses colocam em risco a garantia das diretrizes e princípios assegurados pelo SUS, como acesso, equidade e integralidade na assistência pública de saúde. Logo, a não efetivação do sistema de referência e contra-referências, por exemplo, compromete os princípios da equidade, universalidade e a oferta de soluções às desigualdades nas necessidades de saúde.

No que diz respeito à desigualdade no acesso aos serviços de saúde, é necessária a articulação da ESF com os demais níveis da assistência. A ampliação da cobertura em saúde trazida pela criação e expansão da ESF se mostra insuficiente para a superação das desigualdades no acesso aos diferentes serviços da rede do SUS ${ }^{(21)}$. Assim, essa porta de entrada à rede assistencial apresenta-se bastante fragilizada, o que repercute na saúde de seus profissionais, revelando a complexidade desse nível assistencial na sua atual organização.

Outro desafio está na falta de (re)conhecimento da importância desse modelo assistencial. Apesar da expansão das equipes no território nacional, usuários e gestores mostram pouco conhecimento do funcionamento e diferencial da ESF. Os gestores, por vezes, privilegiam a assistência no modelo tradicional biomédico. Também, observa-se a dificuldade dos gestores na avaliação da qualidade dos serviços de saúde, discutida em outros estudos ${ }^{(21)}$.

Em relação à violência no cotidiano laboral das equipes autores ${ }^{(22)}$ mostram que existe influência de fatores macrossociais na saúde, como a desigualdade urbana, a relação entre acesso à educação e emprego, bem como influência de fatores de natureza contingencial, como o aumento das necessidades e a impossibilidade de satisfazê-las, as mudanças culturais e estruturais das famílias e a redução da capacidade das organizações da sociedade civil em promover a inclusão social. Ainda, o aumento da densidade em áreas pobres e segregação urbana, o culto à masculinidade, fácil acesso a drogas, a 
impunidade, aumento do número de armas de fogo e a dificuldade de expressão verbal dos sentimentos, contribuem para aumentar a violência.

No que se refere à saúde dos profissionais, estudos mostram uma série de situações do cotidiano da ESF que os expõem ao adoecimento e requerem intervenção ${ }^{(8-9,11-12)}$. Entre as ferramentas úteis destacam-se a construção de estratégias coletivas de enfrentamento dos problemas e cargas de trabalho(11) e maior fiscalização nas condições de trabalho.

Com relação aos aspectos/características que contribuíram para redução das cargas de trabalho evidenciou-se a identidade das equipes com o modelo assistencial da ESF, que propicia melhor resolutividade e qualidade na assistência aos usuários, assim como um trabalho mais colaborativo e satisfatório para as equipes.

Observou-se que o trabalho em equipe é capaz de descentralizar a assistência da figura do médico, favorecendo a tomada de decisão coletiva, a autonomia dos diversos profissionais e maior visibilidade de suas atividades ${ }^{(13-14)}$.

A demandaagendada, comofontedereduçãodas cargas de trabalho, também traz questionamentos e retoma a necessidade de estudos sobre a forma de organização e gestão dos serviços da ESF. O SUS introduziu a diretriz do controle social, no entanto a ideia de gestão compartilhada depende de ampla reformulação da mentalidade e da legislação e requer a criação de espaços coletivos, em que as equipes compartilham a elaboração de planos gerenciais e de projetos terapêuticos com os usuários, uma vez que as políticas de humanização têm buscado ampliar o poder dos usuários no cotidiano dos serviços de saúde.

Ainda, lembra-se a necessidade de novas estratégias gerenciais para garantir a qualidade dos serviços públicos de saúde, entre eles a criação de equipes para apoio matricial às equipes de $\mathrm{ESF}^{(4,23-24)}$.

A ESF é, com certeza, uma estratégia inovadora e com grande potencial de contribuição para a universalização do acesso, mas necessita superar inúmeras dificuldades quevêm emergindoao longo de seu processo de implementação e desenvolvimento. Lembra-se que as desigualdades em saúde não são passíveis de ser equacionadas somente no âmbito do setor saúde, pois possuem determinantes políticos, econômicos, sociais e ambientais. perversos níveis de desigualdade, com um sistema de proteção social ainda frágil apesar dos avanços, entre outros problemas, a ESF pode se constituir uma ferramenta capaz de fortalecer a participação comunitária e fortalecimento da consciência sanitária. Para isso, é necessário que sua expansão se faça acompanhada do aparelhamento de suas equipes, dotando-as da capacidade de efetivamente superar o modelo médico-assistencial hegemônico e assumir sua função social, voltandose para a construção de projetos, ações e programas capazes de interferir positiva e efetivamente sobre os determinantes sociais e políticos da saúde e, assim, auxiliar para a efetivação de políticas públicas preconizadas pelo $\mathrm{SUS}^{(2)}$.

\section{CONCLUSÃO}

t Foi possível identificar as fragilidades das equipes e a necessidade de investimentos urgentes para a qualificação da assistência e redução das cargas de trabalho. O estudo revela aspectos da relação entre o trabalho na Saúde da Família em apenas uma das múltiplas regiões do país, contudo, os resultados contribuem para compreensão dos aspectos que aumentam as cargas, e consequentemente elevam o desgaste dos profissionais de saúde na ESF, bem como aspectos que devem ser fortalecidos, permitindo maior proteção ao desgaste e redução das cargas de trabalho, favorecendo o trabalho realizado pelos profissionais.

\section{REFERÊNCIAS}

1. Paim JS. Por um planejamento das práticas de saúde. Ciênc. saúde colet. 1999;4(2):243-9.

2. Campos GWS. Subjetividade e administração de pessoal: considerações sobre modos de gerenciar o trabalho em equipes de saúde. In: Merhy E, Onocko $\mathrm{R}$ (organizadores). Agir em saúde: um desafio para o público. São Paulo: Hucitec; 1997. p.386- 96.

3. Ministério da Saúde (BR). Secretaria de Atenção à Saúde. Departamento de Atenção Básica. Política Nacional de Atenção Básica. Brasília: Ministério da Saúde; 2012.

4. Onocko-Campos RT, Campos GWS, Ferrer AL, Corrêa CRS, Madureira PR, Gama CAP, et al. Avaliação de estratégias inovadoras na organização da atenção primária à saúde. Rev. Saúde Públ. 2012;46(1):43-50.

Numa realidade social como a brasileira, com 
5. Paim JS. Atenção Primária à Saúde: uma receita para todas as estações? Saúde debate. 2012;36(94):343-7.

6. Milstein B, Homer J, Hirsch G. Analyzing national health reform strategies with a dynamic simulation model. Am J Public Health. 2010;100(5):811-9.

7. Laurell AC, Noriega M. Processo de produção e saúde: trabalho e desgaste operário. São Paulo: Hucitec; 1989.

8. Trindade LL, Pires DEP. Implications of primary health care models in workloads of health professionals. Texto Contexto Enferm. 2013;22(1):36-42.

9. Silva MM, Medeiros SM, Cartaxo FJA. Influencia del contexto de trabajo en la salud de los profesionales de enfermería de una unidad de cuidados intensivos en un hospital universitário. Enferm. glob. 2013;12(4):185-97.

10. Bardin L. Análise de conteúdo. Lisboa: Edições 70; 2009.

11. Trindade LL, Lautert L. Síndrome de Burnout entre os trabalhadores da estratégia de saúde da família. Rev Esc Enferm USP. 2010;44(2):274-9.

12. Duarte MLC, Avelhaneda JC, Parcianello RR. A saúde do trabalhador na estratégia de saúde da família: percepções da equipe de enfermagem. Cogitare enferm. 2013;138(2):323-30

13. Marin MJS, Storniolo LV, Moravcik MY. A humanização do cuidado na ótica das equipes da estratégia de saúde da família de um município do interior paulista, Brasil. Rev Latino-Am. Enfermagem. 2010;18(4):763-9.

14. Bonelli MG. Rumo ao interior: médicos, saúde da família e mercado de trabalho. Cad. Saúde Pública. 2009;25(11):2531-2.

15. Anjos RMP, Gianini RJ, Minari FC, Luca AHS, Rodrigues MP. "Vivendo o SUS": uma experiência prática no cenário da atenção básica. Rev. bras. educ. med. 2010;34(1):172-83.

16. Prestes E, Veras R. Educação, qualificação, trabalho e políticas públicas: campos em disputas. Rev. Lusófona Educ. 2009;(14):45-59.

17. Costa GD da, Cotta RMM, Ferreira MLSM, Reis JR, Franceschini SCC. Saúde da família: desafios no processo de reorientação do modelo assistencial. Rev. bras. enferm. 2009;62(1):113-8.

18. Pires DEP, Lopes MGD, Silva MCN, Lorenzetti J, Peruzzo AS, Bresclani ER. Jornada de 30 horas semanais: condição necessária para assistência de enfermagem segura e de qualidade. Enferm. Foco. 2010;1(1):114-8.

19. Pontes APM, Cesso RGD, Oliveira DC, Gomes AMT. A universalidade do SUS: pensamento dos usuários. Esc. Anna Nery. 2009;13(3):500-7.
20. Rosa RB, Pelegrini AHW, Lima MADS. Resolutividade da assistência e satisfação de usuários da estratégia saúde da família. Rev. Gaúcha Enferm. 2011;32(2):34551.

21. Bosi MLM, Pontes RJS, Vasconcelos SM. Dimensões da qualidade na avaliação em saúde: concepções de gestores. Rev. Saúde Públ. 2010;44(2):318-24.

22. Briceño-León R. Urban violence and public health in Latin America: a sociological explanatory framework. Cad. Saúde Pública. 2005;21(6):1629-48.

23. Campos GWS, Domitti AC. Apoio matricial e a equipe de referência: uma metodologia para gestão do trabalho interdisciplinar em saúde. Cad. Saúde Pública. 2007;23(2):399-7.

24. Starfield B. Reinventing primary care: lessons from Canada for the United States. Health Aff (Millwood). 2010;29(5):1030-6. 